\title{
Das Ziel Im Auge
}

\author{
Hans Gnad
}

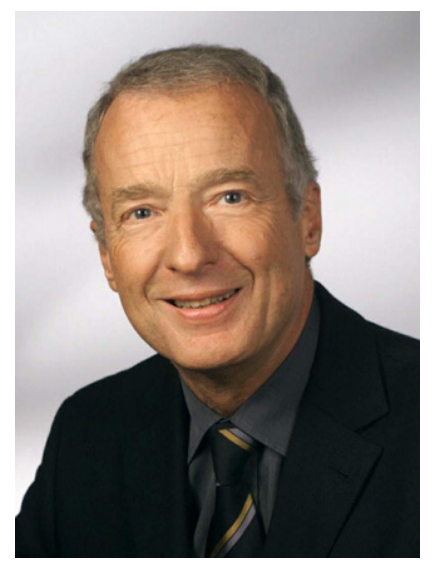

Ausgerechnet das frühere „Noli me tangere“ in der Augenheilkunde, der Glaskörper, war mein erster Berührungspunkt mit meinem späteren Fach.

So wie heute, war auch nach meiner Promotion 1968 das mittelfristige Finden einer Ausbildungsstelle in Wien mit einer verzweifelten Suche verbunden. Das Eintragen auf die Wartelisten der 1. und 2. Univ.Augenklinik war obligat. Das Vorstellen an der Klinik gelang nur bis in die Sekretariate. Von Prof. Nemetz im Hanusch Krankenhaus und von Prof. Fanta in der Rudolfstiftung wurde ich persönlich auf die Aussichtslosigkeit meines Unterfangens hingewiesen. Und Prof. Stepanik, 20 Jahre später mein Vorgänger im KH Lainz, meinte, eher könne er mich an der Klinik unterbringen, bevor ich in Lainz einen Ausbildungsplatz bekäme. Somit war Wien für mich gesperrt und die Suche nach einer Augenstelle erweiterte sich auf ganz Österreich.

\section{Literaturliste beim Verfasser}

Prim. Prof. Dr. H. Gnad ( $\triangle)$

Goldmarkplatz 8, 1130 Wien, Österreich

gnadhans@aon.at
Diese Wartezeit verbrachte ich als Gastarzt am Neurohistologischen Institut bei Prof. Seitelberger. Mit seinem Assistenten H. Bernheimer, dem späteren Vorstand dieses Instituts fuhr ich 1-2 mal in der Woche nach St. Marx, wo wir Rinder- und Schweinebulbi einsammelten, um vor allem die Glaskörperstrukturen histologisch und biochemisch zu untersuchen. Das Hantieren mit dem unantastbaren (und unappetitlichen) Tabubereich des Auges wurde zur täglichen Übung.

Schließlich bot mir der Chef der Univ.-Augenklinik Innsbruck, Prof. Heinz, eine Ausbildungsstelle an, und ich griff erfreut zu. Die Klinik hatte eine sehr überschaubare Größe, das Verhältnis der Mitarbeiter untereinander war im Großen und Ganzen ein sehr freundliches und familiäres.

Ich teilte mein Dienstzimmer mit dem jungen Oberarzt Dr. Franz Orou. Er wurde mein Lehrer, mein Freund, und führte mich in die für einen Anfänger ja völlig fremde Welt der Ophthalmologie ein.

Ein Hobby von Prof. Heinz, und somit ein Schwerpunkt an der Klinik, war die Optik des menschlichen Auges, vornehmlich die objektive Refraktionsbestimmung mittels Skiaskopie, sowie die Strabologie. Dementsprechend gründlich war darin die Ausbildung [25, 30].

In diese Zeit fiel die Geburtsstunde der Fluoreszenzangiographie und der Photokoagulation mittels der Xenon-„Lichtbombe“ der Fa. Zeiss. Erste wissenschaftliche Arbeiten beschäftigten sich mit der Angiographie selbst und mit der von mir entwickelten kinematographischen und videographischen Darstellung der Netzhaut- und Aderhautdurchblutung [5-9, 11, 12, $18,21]$.

Operativer Standard bei der Cataractoperation war die intrakapsuläre Pinzettenextraktion nach fermentativer Zonulolyse, eine Technik, die den heutigen jungen Ärzten bereits wieder völlig unbekannt ist. 
Die Versuche, die Linse durch eine Kunstlinse $\mathrm{zu}$ ersetzen, hatte in den 50er und 60er Jahren zu derart katastrophalen Spätergebnissen geführt, dass davon längst wieder abgegangen worden war.

Üblich war der Graefeschnitt über 160-180 Grad, Wundverschluß mit 3-5 Barraquer Seidefäden, Binoculus für die erste Nacht, tägliche Bettvisite, Spaltlampenuntersuchung nach dem 4. Tag, Aufenthaltsdauer 8-14 Tage, Rasierverbot, Starbrille.

Operiert wurde mit bloßer Hand, Ablationes manchmal mit Zwirnhandschuhen, aber der Gedanke, wie teilweise in Westeuropa oder in den USA schon üblich, Operationshandschuhe zu tragen, ließ die damaligen Chirurgen erschauern.

„Nie könne man mit einem Gummihandschuh das nötige Gefühl für diese delikate Operation aufbringen“ (Heinz). Lupenbrillen zur genauen Sicht waren selbstverständlich, aber „ein Operationsmikroskop zu benützen schien irreal, könne man damit ja das Auge nicht von allen Seiten betrachten“.

Operateur und Assistent standen einander gegenüber. Für den Assistenten bedeutete es eine Auszeichnung dabei sein zu dürfen, und er hatte die Aufgabe, bei der Pinzettenextraktion die Hornhaut zurückzuhalten. Temporäre postoperative Keratopathien prägten den Alltag.

Ablationes wurden am Vorabend der Operation aufgelegt, Netzhautdefekte wurden mit dem Heine Ophthalmoskop eingestellt und und mit Gentianaviolett auf der Bindehaut markiert. Intraoperativ Umstellung des Defektes mit Diathermiekoagulation, Drainage und statt einer Plombe Binoculus. Lochbrille für einige Wochen.

Zwischenzeitlich hatte Dr. Orou die Augenklinik in Zürich kennengelernt und empfahl mir das Gleiche im Rahmen eines Sommerurlaubs. Nach 4 Wochen Gastarzttätigkeit war ich von der Klinik so begeistert, dass ich mich um eine Assistentenstelle bewarb und diese nach fast 3 Jahren in Innsbruck (inkl. der Gegenfächer) 1971 antreten konnte.

Zürich war ein Highlight in Ophthalmologie. Der Schweiz entsprechend, gediegene Ausstattung, konservativ im Geiste von Vogt und Amsler, machten Prof. Witmer als deren Nachfolger und sein Oberarzt, Prof Klöti diese Klinik zu einem weltbekannten Zentrum in der Entwicklung innovativer Operationen und neuer Therapien.

Lehre war kein leeres Wort, junge Assistenten wurden tatsächlich operativ und konservativ ausgebildet, mussten neue Literatur referieren und wurden $\mathrm{zu}$ wissenschaftlicher Arbeit angehalten. Ein Traum für interessierte junge Kollegen.

Elektroretinographie [14], Neuroophthalmologie, Entstehung des Keratokonus [13], Glaukom-, Uveitis[11] und genetische Forschung [10] waren konservative Schwerpunkte.

Operativ tat sich für mich eine völlig neue Welt auf. Operationsmikroskop, Handschuhe, indirekte
Ophthalmoskopie bei der Ablatiooperation waren Standard.

Bei der Cataractoperation wurde das Auge wie heute ab externo eröffnet, Nylonnähte wurden vorgelegt, und neben der Pinzettenextraktion hatte sich die Kryoextraktion der Linse etabliert.

Nach genügender operativer Ausbildung durch die Lehroberärztin, Frau Prof. Martenet, gab mir Prof. Witmer die Möglichkeit, für 3 Monate in Madurai in Südindien meine operative Routine $\mathrm{zu}$ erwerben. Ausgestattet mit dem Geld einer Schweizer Stiftung und einem doppelten Set neuer Grieshaber Instrumente konnte ich in diversen Eye camps mit einem Operationskatalog nach Zürich zurückkommen, der u. a. 420 Cataractoperationen enthielt.

Prof. Klöti war ein unglaublich erfahrener Ablatiooperateur und lehrte mich Cerclagen mit frisch entnommener Fascia lata, die Anwendung der von ihm entworfenen Silberklemme bei zentralen Ablationes mit Maculaloch, die Anbringung radiärer oder longitudonaler Plomben, die Lochlokalisation mit dem Bonnoskop (einem Vorläufer der binokularen indirekten Ophthalmoskopie) und die Kryoapplikation auf die Netzhautdefekte.

Ein unschätzbar weiterer Pluspunkt war, dass genau in meiner Züricher Zeit Prof. Klöti die Vitrektomie entwickelte. Diese völlig neue Operation dauerte naturgemäß stundenlang und war immer für die späten Nachmittage angesetzt. Für manche Assistenten langweilig, war es für mich eine willkommene Gelegenheit diese Operation ab ovo kennenzulernen [17, 22, 23].

1973 traf ich im Rahmen einer gemeinsamen Tagung der DOG und ÖOG Prof. Hruby in Würzburg, und er fragte mich nach meinem Vortrag „Über die Ursache der ausgelöschten Aderhautfluoreszenz am hinteren Pol“, warum ich denn nicht nach Wien käme.

Ich war knapp vor Beendigung meiner Ausbildungszeit und nahm sein Angebot, an die 1. Univ.Augenklinik zu kommen, sehr gerne an.

Der Wiedereintritt in Wien 1974 war alles andere als problemlos. Als ich mich zum vereinbarten Zeitpunkt zum Arbeitsantritt meldete, wurde mir mitgeteilt, dass die für mich vorgesehene Stelle irrtümlich bereits wieder besetzt worden war und ich mich doch an der 2. Augenklinik vorstellen möge. Der Schreck war groß, und an der 2. Augenklinik war natürlich auch keine Stelle frei. Letztlich begann ich für ein Jahr als Gastarzt an der I. Augenklinik zu arbeiten und wurde (damals als Vater von 2 kleinen Kindern) aus dem Etat der Besonderen Gebühren unterstützt.

Die Wiener Klinik war damals in weiten Bereichen ähnlich dem Niveau der Innsbrucker Klinik. Ich wurde Assistent auf der Männerstation bei Doz. Kutschera, der ebenfalls nach einem Besuch der Züricher Klinik, sich bemühte Mikroskop, Handschuhe und indirekte Ophthalmoskopie einzuführen. Die Gewohnheit, bei Operationen einander gegenüber $\mathrm{zu}$ stehen war so eingefahren, dass auch das erste Mikroskop die Okulare visavis angebracht hatte und als sich schließlich das 
Operieren von oben durchsetzte, begannen für den Assistenten schwere Zeiten, da er dann nur durch extreme Verkrümmung des Rückens an der Operation teilnehmen konnte.

Zwei Disziplinen waren der Züricher Klinik überlegen: Echographie und die Behandlung der diabetischen Retinopathie. Beide wurden im Rahmen von Spezialambulanzen von Doz. Freyler aufgebaut und geleitet. Freyler war damals Oberarzt auf der Chefstation.

Nach einem Jahr auf der Männerstation wurde ich gemeinsam mit Klemen Freylers Assistent. Damit verbunden war aber die Aufgabe, ein- bis zweimal wöchentlich in Prof. Hrubys Ordination als Schriftführer zu agieren. Von seiner abgedunkelten Kammer aus diktierte er Untersuchungsergebnisse, z. B. den Javalwert. Und wenn Hruby dann skiaskopierte und den Javalwert abfragte, ergaben sich kabarettartige und nicht wiedergebbare Diskussionen über die Höhe oder Achse der Hornhautkrümmung.

Waren Orou in Innsbruck und Witmer in Zürich meine Mentoren, so fand ich in Freyler einen Vorgesetzten und Freund, dem ich lebenslang freundschaftlich und loyal verbunden bleiben werde.

Kutschera wurde 1977 Chef der Augenklinik in Salzburg, Freyler übersiedelte auf die Männerstation und ich wurde Oberarzt auf der Chefstation. Die Chefstation hatte ein kleines Zimmer der allgemeinen Gebührenklasse mit 8 Betten, und das war der Einstieg in die operative Eigenverantwortlichkeit. Verbunden mit dieser Funktion war aber die Tatsache, dass Prof. Hruby von seinem Oberarzt erwartete, ihn täglich bei der Visite zu begleiten. Und täglich hieß jahrelang wirklich täglich, nämlich an Wochenenden genauso wie an Feiertagen, zu Weihnachten oder zu Ostern. Ging er auf Urlaub, musste ich die Stellung halten, und wenn ich auf Urlaub gehen wollte, war das nur eingeschränkt möglich, weil er sonst seinen gewohnten Begleiter vermisst hätte.

Für mich tat sich schrittweise ein weites Feld auf. Hruby erlaubte die Anschaffung des Klöti-Vitrektomiegeräts der Fa. Örtli, und das Gerät war damals so heikel in der Bedienung und Reinigung, dass ich für einige Zeit das Alleinbenützungsrecht in Anspruch nahm. Mit zahlreichen Kollegen ergaben sich wissenschaftliche Synergien (Freyler, Klemen, Skorpik, Menapace, Radda, Prskavec, Drobec, Paroussis, 26, 31, 32, $33,34,44)$.

1981 wurde Prof. Hruby emeritiert, Freyler übernahm, zunächst für ein Jahr supplierend, die I. Univ.Augenklinik und ich habilitierte mich mit dem Thema: „Die Vitrektomie via pars plana. Eine histologische Studie und Analyse klinischer Ergebnisse“.

1982 wurde Freyler zum Ordinarius bestellt, ich wurde sein Vertreter, hatte die Diensteinteilungen über und die zahlreichen ausländischen Gastärzte der American Acadamy zu betreuen. Für 2 Jahre leitete ich die Frauen-, und dann, bis zu meinem Ausscheiden aus der Klinik 1988, die Männerstation.
Noch 1979 wurde ich von der Fa 3M zu einem Kongress der ASCRS nach Singapore eingeladen. Für mich geographisch, vor allem aber fachlich, ein unglaubliches und begeisterndes Neuland. 20 Jahre nach dem Ende der ersten Vorderkammerlinsenära hatte vor allem Binkhorst an der Idee des Linsenersatzes festgehalten. Statt im Kammerwinkel wurde die Linse an der Iris fixiert und kam, von uns konservativen Ophthalmologen förmlich unbemerkt, in einer Unmenge von Variationen vor und hinter der Iris platziert, in Holland, England, den USA und auch in Russland, zum Einsatz.

Schon 1980 konnten wir eigene erste Frühergebnisse nach Implantation künstlicher Linsen publizieren [36]. Die Implantation erfolgte in den ersten Fällen nach intrakapsulärer Kryoextraktion. Die Operation war delikat, Healon war noch nicht bekannt, die Positionierung der Linse und die Nahtfixation an der Iris musste unter einer Luftblase erfolgen. Ließ sich die Luftblase nicht halten, wurde von der Implantation abgesehen, um die Gefahr des zystoiden Makulaödems hintanzuhalten.

In der Folge ging die Entwicklung rasant weiter. Der Klöti-Stripper erwies sich als große Hilfe für die schon 1982 erfolgte routinemäßige Umstellung der Extraktion auf die ECCE [26]. Healon erleichterte die Operation und 1983 begannen wir mit der Phakoemulsifikation. Die erste Phakomaschine von Cooper Vision konnte die Vorderkammer nicht immer stabil halten und die Furcht, die hintere Linsenkapsel zu verletzen, war groß. Freyler und ich flogen mehrmals zu Prof. Nover und Neuhann nach Mainz, um die Phakotechnik zu erlernen. Häufig wurde die Operation mit Phako begonnen und intraoperativ auf ECCE wieder umgestellt. Von meinen langjährigen Mitarbeitern, R. Menapace, P. Paroussis, F. Prskavec,und M. Grasl war es Chr. Skorpik, dem als Erstem an der Klinik eine durchgehende Phakoemulsifikation gelang. Gleichzeitig erfolgte damals der Wechsel von der Iriscliplinse auf die Hinterkammerlinse.

Am Beginn der 80er Jahre unterlag auch die Netzhautchirurgie einem raschen Wandel. Luft, SF6 Gas o. dgl. wurde nach Vitrektomie insuffliert [46, 64, 66]. Healon, der vermeintlich ideale Glaskörperersatz, erwies sich als völlig ungeeignet und kontraindiziert [54], und bei Zivojnovic in Rotterdam erlernten wir die Silikonölimplantation [58, 68, 124].

Ich erinnere mich, dass wir anfangs die Silikonölchirurgie akrobatisch ausarten ließen, zum kompletten Flüssigkeitsaustausch gegen Silikonöl wurde der Patient in Bauchlage umgebettet und ich lag auf dem Fussboden darunter und implantierte das im flüssigkeitsgefüllten Bulbus aufsteigende Öl. Mit der Einführung der „Staubsaugerkanülen“ war diese Periode dann wieder beendet.

Es blieb eine Zeit höchster Aktivität und rascher Neuerungen. Die refraktive Chirurgie, von „seriösen“ Kliniken verachtet, war nicht mehr zu übersehen. 
Im Rahmen von Kursbesuchen, oft über ein verlängertes Wochenende in den USA, bei Barraquer in Bogota oder bei Krumeich in Bochum, erlernten wir die radiäre Keratotomie, die Keratomileusis, die Epikeratophakie mit frischen oder lyophilisierten Lentikeln [79, 101, 106], Techniken, die längst wieder aufgegeben worden sind, aber den Weg zur Laserchirurgie vorzeichneten.

1983/84 wurde ich zum Präsidenten der Ophthalmologischen Gesellschaft in Wien gewählt und hatte die Gelegenheit, eine Reihe prominenter ausländischer Gäste, wie Jörg Dräger, Holger Busse, Peter Kroll, Peter Speiser, Jules Francois, Christoph Huber, Reljy Zivojnivic und meinen Lehrer Rudolf Witmer als Vortragende einzuladen.

Um ihnen zu danken, organisierten wir die Nachsitzungen damals regelmäßig in Grinzing beim Heurigen „Oppolzer“ in der Himmelstrasse.

Von 1975 bis 1985 betreute ich im Rahmen einer Konsiliararzttätigkeit einmal wöchentlich die entwicklungsgestörten Kinder im neu aufgebauten Pavillon von Prof. Rett im Neurologischen Krankenhaus Rosenhügel [30, 85]. Es war ein Einblick in eine Welt, in der man Wunder nicht so einfach mit dem Skalpell bewerkstelligen konnte.

W. Kulnig hat diese Aufgabe danach dankenswerterweise übernommen.

Es war nicht mein Ziel, als Oberarzt in Pension zu gehen. Ich habe heute noch die Kopie eines Briefes, den Prof. Witmer 1973 anlässlich meiner Übersiedlung nach Wien Prof. Hruby geschrieben hatte, in dem er erwähnt, dass ich Interesse hätte, einmal meine eigene Abteilung zu leiten, z. B. die im Krankenhaus Lainz.

Bis es soweit kommen sollte, waren aber auch andere Abteilungen zur Besetzung ausgeschrieben. Im Wilhelminenspital sollte eine Abteilung wieder errichtet werden.

Mein Freund A. Thaler und ich bemühten uns darum, in der Nacht vor der entscheidenden Sitzung des Sanitätsrates wurde das Vorhaben von Prof. Stacher aber aus finanziellen Gründen wieder abgeblasen.

Die Barmherzigen Brüder planten die Reaktivierung der ehemaligen Augenabteilung und die Grazer Univ. Augenklinik war ausgeschrieben, für beide Abteilungen habe ich mich beworben, im Grunde aber wollte ich nach Lainz.

1987 ging Prof. Stepanik in den Ruhestand und im Frühjahr 1988 trat ich seine Nachfolge an. Prof. Stepanik war ein akribisch forschender, international anerkannter Glaukomexperte, der Operationssaal war nicht sein Steckenpferd. Ich musste also anfangs aus eigenem viele Dinge einbringen, um den operativen Standard, den wir im AKH längst erreicht hatten und der internationales Ansehen hatte, in Lainz herzustellen. Das reichte vom Kopierapparat, über Operationsstühle, der Phakomaschine und den Vitrektomiegeräten bis hin zum Autoklaven und dem Klimagerät im Operationssaal.
Damit war es mir möglich, vom ersten Tag weg, das gleiche operative Niveau zu halten, wie ich es von der Klinik gewohnt war.

Es war ein Neubeginn, der auch nur mit Hilfe der Kollegen, die von der Klinik mit mir kamen, gelingen konnte:

M. Juchem (die viel zu früh wieder von uns ging), R. Schranz (den ich in Augenheilkunde geprüft hatte und der letztlich mein Partner in der Ordination wurde), W. Scheidel, J. Funder, Chr. Dolezal und F. Skorpik.

2 Oberärzte aus der Stepanikriege, I. Pronai und Z. Stein blieben gerne, und sehr rasch bildeten wir ein Team von 13 Ärzten mit dem es eine Freude war, gemeinsam die Abteilung zu einem operativen Zentrum aufzubauen.

Waren es an den früheren Kliniken die Ärzte Orou, Witmer und Freyler, die mir hilfreich zur Seite standen, so traf ich in Lainz auf (m)eine Martina. Martina war schon Chefsekretärin bei Stepanik, war organisatorisch und fachlich, vor allem aber menschlich derart überragend, dass sie das Herz der gesamten Mannschaft bildete.

Als Phakozentrum veranstalteten wir Phakokurse im Haus, wurden aber auch nach Oslo, Prag, Bratislava und Brünn zum Demonstrieren dieser Technik eingeladen.

Fercher, Drexler, Hitzenberger und Schmetterer arbeiteten mit unseren Patienten an der Laser Doppler Interferometrie, der Grundlage für das heutige OCT.

Die Keratoplastikfrequenz erforderte den Aufbau einer eigenen Hornhautbank, die von meiner Oberärztin N. Karnik geleitet wurde und im regen Austausch mit der Hornhautbank im AKH stand.

M. Rubey und F. Skorpik fanden ihren Schwerpunkt in schwierigen vitreoretinalen Engriffen und erlernten sehr rasch den Umstieg von der Aufleglinse auf das Biom.

Juchem, eine geniale Operateurin mit grenzenloser Aufopferung forcierte die No-stitch-Technik, sammelte große Erfahrung mit den aufkommenden Falt- und Multifokallinsen und war eine geduldige Lehrerin im Operationssaal.

Wir eröffneten ein „Ludwig Boltzmann Institut für intraokulare Kunstlinsen“, gründeten den gemeinnützigen Verein „Gesunde Augen-Gutes Sehen“ und dotierten ihn aus Spenden sowie einem Teil der besonderen Gebühren.

Apropos, in diese Zeit fiel auch der von mir, A. Thaler und vor allem von P. Heilig vehement geführte Kampf für gesunde Augen und gutes Sehen, nämlich unsere Initiative gegen die geplante Aktion „Licht am Tag“. Eine österreichweite Umfrage bei allen Augenärzten unterstützte nahezu einhellig unsere ablehnende Haltung, aber letztlich war es eher ein politischer Zufall, dass die Lichtpflicht wieder aufgehoben wurde.

Wo viel Licht ist, ist auch viel Schatten. Ein dunkles Kapitel in fast allen Pavillons von Lainz bildete der bauliche Zustand. Es war wie in alten Klinikzeiten. Ungenügende sanitäre Einrichtungen, große Kranken- 
säle, und ein Operationssaal, der diesen Namen nicht verdiente. Seit der Errichtung des Krankenhauses 1913 waren de facto keine Verbesserungen durchgeführt worden. Riesige südseitige Fenster ließen im Sommer das Thermometer im Operationssaal auf über $35 \mathrm{Grad}$ ansteigen.

Jahrzehntelang wurden Abteilungen damit vertröstet, dass sehr bald ein gemeinsamer Operations- und Ambulanzbau errichtet werde. Bis heute ist dieser Plan nicht umgesetzt worden. Die Lage schien aussichtslos.

Vor allem aber wussten wir an der Augenabteilung, dass sich aus den vorhandenen Räumlichkeiten keine neuen Operationssäle bilden ließen. Von dem uns sehr hilfreich zur Seite stehenden damaligen Stadtrat K. Rieder mussten wir auch zur Kenntnis nehmen, dass die finanziellen Mittel nicht ausreichen würden. Es war mein Oberarzt A. Nowak, der die Idee von 2 in den Hof gestellten, am Jugendstilpavillon angedockten Operationscontainern hatte, und sie als Provisorium vor dem Denkmalamt deklarierte.

Und als Geldgeber bzw. Sponsoren hatten wir die Firmen Askin mit H. Lugbauer und Pharmacia mit H. Blaha, deren Produkte wir ohnedies verwendeten und damit langsam unsere Schulden tilgten. Gedacht, getan, die gesamte Abteilung übersiedelte für fast 2 Jahre in andere Pavillons, Bettentrakt, Ambulanz und Operationssaal voneinander getrennt. Es war eine mühsame Zeit, aber es lohnte sich.

1995, 7 Jahre nach meinem Beginn in Lainz wurde die gesamte neu gestaltete Abteilung feierlich eröffnet. Nun hatten wir auch baulich eine Vorzeigeklinik in einem Jugendstilambiente und von der Gediegenheit her erinnerte sie mich an die Züricher Klinik. Ein moderner Operationstrakt mit Schleuse, Sterilisationsraum, 2 gleichwertigen Operationssälen mit Deckenstativen, eine Sehschule, kleine moderne Krankenzimmer und eine große Bibliothek, die auch als Besprechungs- und Vortragsraum allen Mitarbeitern genügend Platz bot, ließen fast keine Wünsche mehr offen.

1997-1999 hatte ich die Ehre, die Präsidentschaft der ÖOG zu übernehmen. Bei den Tagungen in Bre- genz und St. Pölten haben wir, dem Wunsch vieler Kolleg(inn)en entsprechend, damit begonnen, Fortbildungsmodule in die Veranstaltung zu integrieren. Diese wurden sehr gut angenommen und deshalb bis heute beibehalten.

Der Vorstand der ÖOG wurde durch die Aufnahme von Mittelbauvertretern erweitert.

1999 tagte auch die ESCRS im Kongresszentrum in der UNO-City in Wien. Von einem in Eigeninitiative erstandenen Excimer Laser, den ich im neuen Operationssaal in Lainz für einige Jahre aufgestellt hatte, übertrugen wir live eine von T. Neuhann durchgeführte Lasik-Operation in den Hörsaal.

20 Jahre nach meinem Start in Lainz konnte ich 2007 eine perfekt funktionierende, gut ausgebaute $\mathrm{Au}$ genabteilung mit einem hervorragendem Team übergeben.

Dieses Ziel war erreicht.

Der Weg war mühsam, aber unglaublich schön und befriedigend.

2000 haben C. Faschinger et al. die Organisation „Sehen ohne Grenzen“ ins Leben gerufen. Seither nehme ich, wann immer sich die Gelegenheit dazu ergibt, an den Eye camps in der Dritten Welt teil. Mit dem tragbaren CataRhex Phakogerät von Örtli im Handgepäck, führten uns zahlreiche chirurgische Expeditionen nach Namibia, Sambia, Simbabwe und Nepal.

Faschinger gelang es, vor allem in Namibia, eine hinreichende ophthalmologische Infrastruktur aufzubauen, sodass bei den jeweiligen Camps - abgesehen von den räumlichen Verhältnissen - die hygienischen Bedingungen am Auge selbst, durchaus unseren Standards entsprechen.

Die Visiten bei diesen Patienten am nächsten Morgen entschädigen für die Mühen des Vortags. Singend und tanzend wird die neue Sicht auf ein förmlich neues Leben begrüßt.

Ruhe kann noch warten.

Interessenkonflikt $\mathrm{H}$. Gnad gibt an, dass kein Interessenkonflikt besteht. 